\title{
Simple Electronic Speckle Pattern Shearing Interferometer with a Holographic Grating as a Shearing Element
}

\author{
Emilia Mihaylova \\ Technological University Dublin, emilia.mihaylova@tudublin.ie \\ Izabela Naydenova \\ Technological University Dublin, izabela.naydenova@tudublin.ie \\ Suzanne Martin \\ Technological University Dublin, suzanne.martin@tudublin.ie
}

See next page for additional authors

Follow this and additional works at: https://arrow.tudublin.ie/cieocon2

Part of the Optics Commons

\section{Recommended Citation}

Mihaylova, E., Naydenova, I. \& Martin, S. (2005). Simple electronic speckle pattern shearing interferometer with a holographic grating as a shearing element. SPIE Proceedings of the International Conference Optical Systems Design, 5962. doi:10.1117/12.625163

This Conference Paper is brought to you for free and open access by the Centre for Industrial and Engineering Optics at ARROW@TU Dublin. It has been accepted for inclusion in Conference Papers by an authorized administrator of ARROW@TU Dublin. For more information, please contact arrow.admin@tudublin.ie, aisling.coyne@tudublin.ie,gerard.connolly@tudublin.ie.

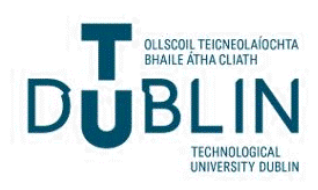




\section{Authors}

Emilia Mihaylova, Izabela Naydenova, Suzanne Martin, and Vincent Toal

This conference paper is available at ARROW@TU Dublin: https://arrow.tudublin.ie/cieocon2/31 
2005-01-01

\section{Simple electronic speckle pattern shearing interferometer with a holographic grating as a shearing element}

Emilia Mihaylova

Dublin Institute of Technology, emilia.mihaylova@dit.ie

Izabela Naydenova

Dublin Institute of Technology, izabela.naydenova@dit.ie

Suzanne Martin

Dublin Institute of Technology, suzanne.martin@dit.ie

Vincent Toal

Dublin Institute of Technology, vincent.toal@dit.ie

\section{Recommended Citation}

E. Mihaylova, I. Naydenova, S. Martin, V. Toal, Simple electronic speckle pattern shearing interferometer with a holographic grating as a shearing element, SPIE proceedings of the International conference Optical systems design, Vol. 5962, 596226, 2005.

This Conference Paper is brought to you for free and open access by the Centre for Industrial and Engineering Optics at ARROW@DIT. It has been accepted for inclusion in Articles by an authorized administrator of ARROW@DIT. For more information, please contact

yvonne.desmond@dit.ie, arrow.admin@dit.ie. 


\title{
Simple electronic speckle pattern shearing interferometer with a holographic grating as a shearing element
}

\author{
Emilia Mihaylova, Izabela Naydenova, Suzanne Martin, Vincent Toal \\ Centre for Industrial and Engineering Optics \\ Dublin Institute of Technology, Kevin Street, Dublin 8, Ireland \\ e-mail: emilia.mihaylova@dit.ie, izabela.naydenova@dit.ie, suzanne.martin@dit.ie, \\ vincent.toal@dit.ie
}

tel: 3531402 4702; fax: 35314024988

\begin{abstract}
An optical set-up for electronic speckle pattern shearing interferometry (ESPSI) using a photopolymer diffractive optical element as a shearing element, is presented. A laser beam illuminates the object at an angle to the normal to the object surface. The holographic diffraction grating is placed in front of the object. The zero and the first order of diffraction form the image and the sheared image of the object. The images are imaged onto the CCD camera, whose optical axis coincides with the normal to the object surface. The field of view is limited only by the dimensions of the photopolymer plate. The photopolymer diffractive element is characterised by low level of light scatter and diffraction efficiency of $60 \%$. The simplicity of the proposed new shearing interferometer is manifested by the extremely small number of components required - a coherent light source, a holographic optical element and a CCD camera.
\end{abstract}

KEYWORDS: shearography, shearing interferometry, holographic grating, HOE

\section{INTRODUCTION}

Electronic speckle pattern shearing interferometry (ESPSI) enables direct measurements of displacement derivatives to be made with variable sensitivity ${ }^{1-11}$. A common approach to generate two sheared images of the object in ESPSI systems is to use a Michelson interferometric optical set-up. The images are generated via the two mirrors and the shear is introduced and controlled by tilting one of the mirrors. The idea of using a holographic grating for shearing of the two images in speckle shearing interferometry is an attractive alternative to the other shearographic systems ${ }^{5-7}$ as it makes for a very simple system.

We suggest a new application of photopolymer holographic gratings in a ESPSI optical set-up. Self-processing acrylamide based photopolymer ${ }^{12}$ is used as a recording medium for recording holographic gratings. The optimized photopolymer material gives good diffraction efficiencies up to $94 \%$ for an exposure of $80 \mathrm{~mJ} / \mathrm{cm}^{2}$.

\section{EXPERIMENTAL SETUP AND PRINCIPLE}

Figure 1 shows the scheme of the proposed ESPSI system using a photopolymer holographic grating as a shearing element. A Helium-Neon laser, with wavelength $633 \mathrm{~nm}$ and output power of $20 \mathrm{~mW}$, is used as the light source. The laser beam illuminates the object at an angle $\theta$ to the normal to the object surface. 
The shearing element is the grating. It is made holographically by the interference of two plane waves. A set of holographic gratings with spatial frequencies of 200 lines/mm, 350 lines/mm, and 500 lines/mm were recorded using a frequency doubled $\mathrm{CW} \mathrm{NdYV_{4 }}$ laser $(\lambda=532 \mathrm{~nm})$. The grating frequency and the distance of the grating from the test object are chosen so that the angle of diffraction $\delta$ (Fig. 2) is large enough to avoid overlapping of the first order and zero order of the diffracted wavefronts.

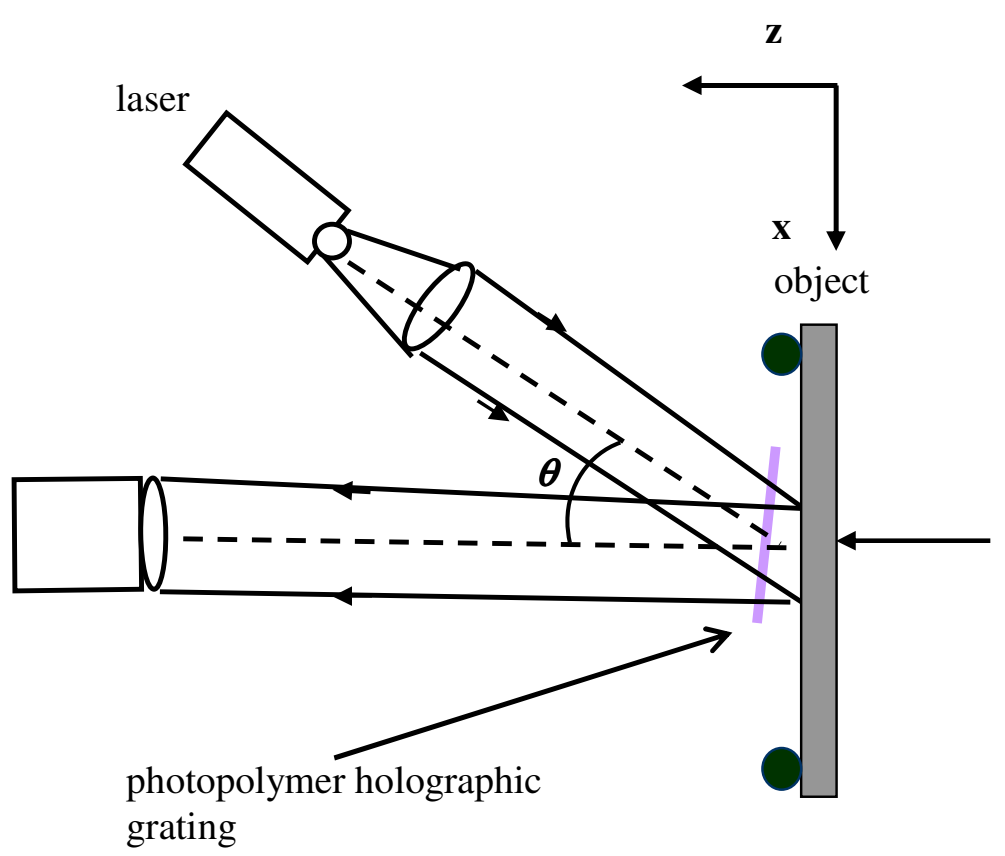

Fig. 1. An optical set-up of the ESPSI system with one photopolymer grating in front of the object

The acrylamide based photopolymer, which is self-developing, was used as the photosensitive medium. The layers were approximately $100 \mu \mathrm{m}$ thick. Recording time and intensity were $20 \mathrm{~s}$ and $3.5 \mathrm{~mW} / \mathrm{cm}^{2}$ respectively. The diameter of these gratings is $40 \mathrm{~mm}$. Diffraction efficiency in the +1 order is $60 \%$. One of the advantages of this material is that characteristics such as diffraction efficiency, thickness (which controls selectivity), slant angle, diameter and reconstruction wavelength and angle can all be chosen to suit the specific application. The photopolymer used is characterized by low scattering and this is important when the imaging properties of the optical system are of concern.

The grating is placed in front of the object at a distance $\Delta z$. The grating divides the illuminating wave into two diffracting waves, which illuminate the object. The two reflected waves form the image and the sheared image on the image plane. These are sheared by the amount $\Delta x$. The shear $\Delta x$ is related to $\Delta z$, the diffraction angle and the illumination angle:

$$
\Delta x=\Delta z|\tan (\theta+\delta)-\tan \theta|
$$

where $\theta$ is the angle of incidence of the light on the grating, and $\delta$ is the angle between the directions of the zero and the first diffracted orders (Fig. 2). The rotation of the grating around its central axis, parallel to y-axis, leads to slight off-Bragg angular adjustment and decrease in the intensity of the first order thus offering the possibility for fine adjustment of the intensities of the zero (the image) and the -1 order of diffraction (the sheared image). 


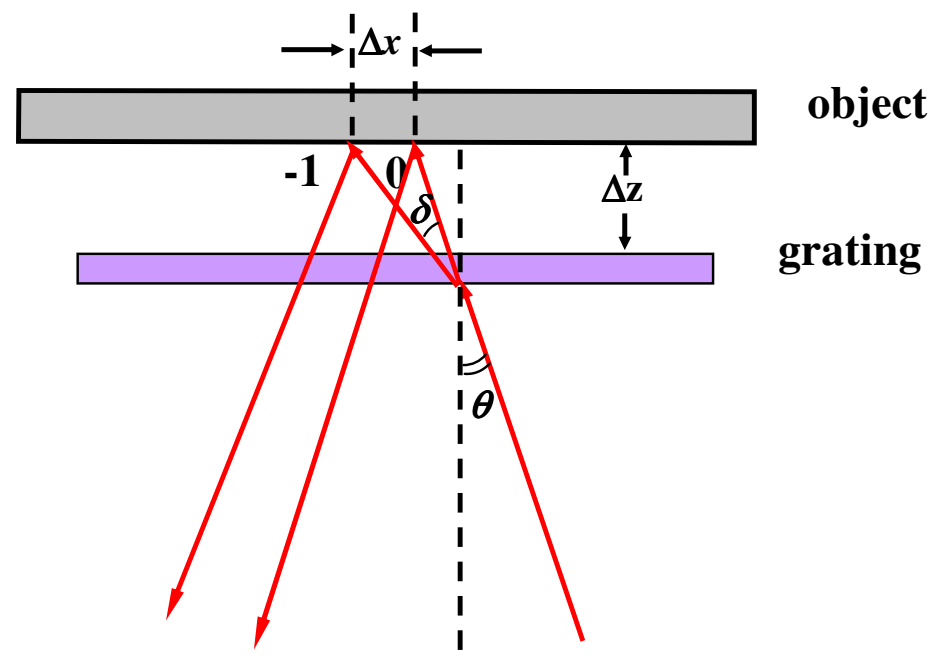

Fig. 2. Mechanism of the wavefront shearing with one holographic grating in front of the object

The two sheared imaged can be viewed in a specific direction only. Therefore, the influence of the scattered light from higher orders has been found to have no influence on the quality of the fringe patterns. Once the optical systems has been aligned and the fringe pattern formed, phase-shifting can be implemented by an in-plane translating of the grating $8,13,14$. The translating of the grating sideways makes the irradiance of the interferogram to vary sinusoidally with time and thus a real-time heterodyne phase detection can be done ${ }^{13}$.

\section{RESULTS AND DISCUSSION}

Figures 3 and 4 show the results from the test of the ESPSI system described above. The ESPSI system is controlled by software ${ }^{15}$. A filter with a $3 \times 3$ window was used to reduce the speckle noise in the images. Fringe patterns presented in Figure 3 were recorded during cooling of an aluminium tin filled with hot water. The fringe pattern characteristic of the derivative of the displacement of the deformed object is displayed on the computer monitor.
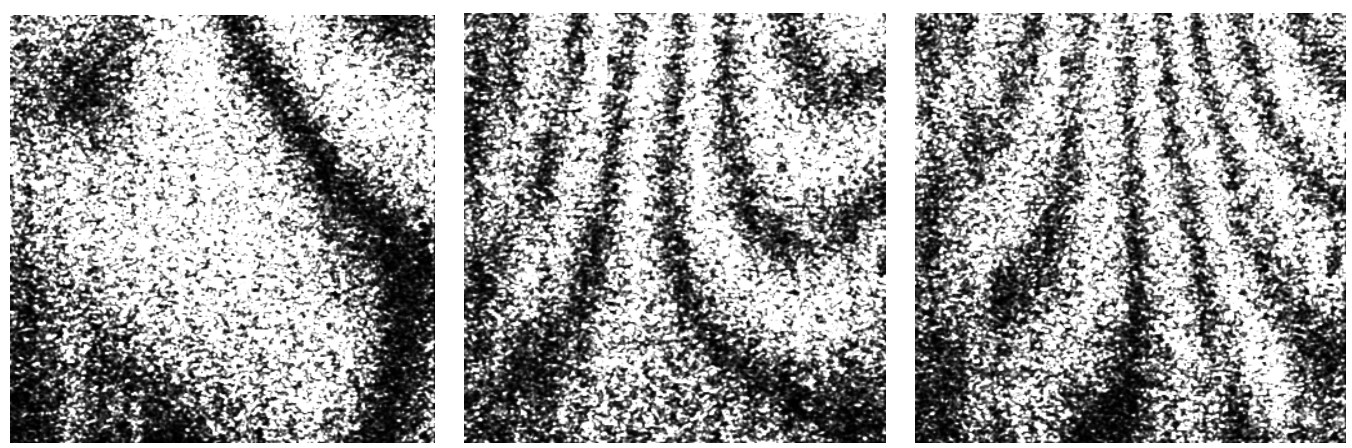

Fig. 3. ESPSI fringes in aluminium tin filled with hot water recorded during cooling with ESPSI system with one holographic grating (frequency $350 \mathrm{l} / \mathrm{mm}$ ) in front of the object: a) at the beginning of data acquisition; b) after $3 \mathrm{~s} ; \mathrm{c}$ ) after $6 \mathrm{~s}$. The shear $\Delta x$ $=6 \mathrm{~mm}$. The field of view is $14 \mathrm{~mm} \times 14 \mathrm{~mm}$. 
Fringe patterns presented in Figure 4 were recorded during pure bending of an polyvinylchloride (PVC) beam with following dimensions: length $-L=130 \mathrm{~mm}$; width $-d=6 \mathrm{~mm}$ and height $-h=27 \mathrm{~mm}$. The deflection was introduced using a vernier support and a step of $1 \mu \mathrm{m}$.
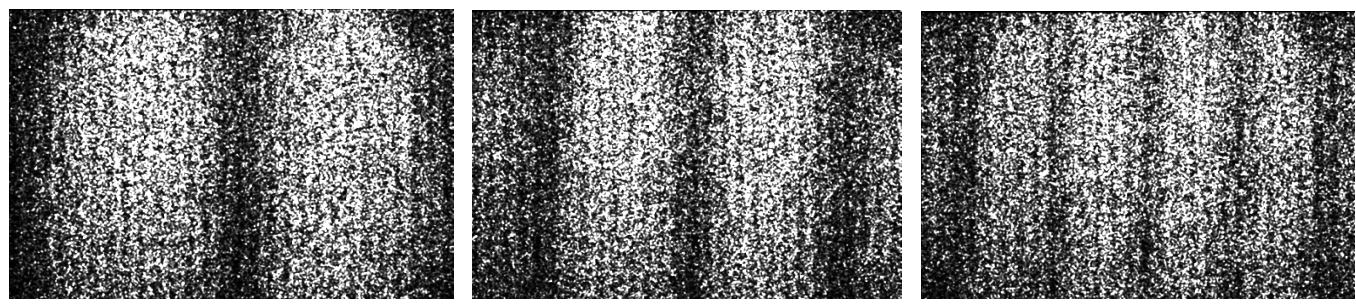

Fig. 4. ESPSI fringes on PVC during pure bending under deflection of: $5 \mu \mathrm{m}$; b) $10 \mu \mathrm{m}$; $) 15 \mu \mathrm{m}$, recorded with ESPSI system with one holographic grating (frequency $200 \mathrm{l} / \mathrm{mm}$ ) in front of the object. The shear is $\Delta x=6 \mathrm{~mm}$. The field of view is $30 \mathrm{~mm} \mathrm{x}$ $20 \mathrm{~mm}$.

Easy control of the shearing amount is important because it determines the overall sensitivity of the system. In this case the distance between the grating and the object controls the size of the shear (Fig. 2). The increase of the amount of shear leads to an increase of the sensitivity of the system. When the distance between the grating and the object is kept constant the shearing amount can be changed by utilizing diffraction gratings with different spatial frequencies. The higher is the spatial frequency of the grating the bigger is the amount of the shear. The spatial frequency of the gratings influences also the contrast of the obtained ESPSI fringes. The size of the field of view depends only on the size of the holographic grating (photopolymer plate) used. The shear in the ESPSI system with one holographic grating in front of the object could be applied in any direction in the plane $(x y)$ by rotating the grating around its normal.

\section{CONCLUSIONS}

The electronic speckle pattern shearing interferometer (ESPSI) presented in this paper is a very simple system having a minimum of components. The holographic grating, recorded using a self-developing acrylamide based photopolymer material, is used to shear the two images. The distance between the grating and the object can be used to control the amount of the shear. The ESPSI system described above is compact and offers a simple way to introduce discrete shear steps between two images. Another advantage of this system is that it is easy to change the shearing direction by rotating of the grating around its normal. Advantages include also the low cost of the system and the potential to use large apertures. The proposed system could be used in a phase-shifting mode.

\section{ACKNOWLEDGEMENTS}

Acknowledgements are made to Technological Sector Research Programme Strand III supported by the Irish Government. Emilia Mihaylova and Izabela Naydenova would like to thank Arnold F. Graves Scholar Programme and FOCAS at Dublin Institute of Technology.

\section{REFERENCES}

1. C. Joenathan C. and Torroba R., "Simple electronic speckle shearing pattern interferometer", Opt. Lett. 15 (20), 1159-1161 (1990).

2. R. S. Sirohi, "Speckle methods in experimental mechanics", in Speckle Metrology, Ed. R. S. Sirohi, Mercel Dekker, New York (1993).

3. Y. M. He, C. J. Tay, H. M. Shang, "Digital phase-shifting shearography for slope measurement", Opt. Eng. 38 (9), 1586-1590 (1999).

4. H. M. Shang, Y. Y. Hung, W. D. Luo, F. Chen, "Surface profiling using shearography", Opt. Eng. 39(1), 23-31 (2000).

5. P. Hariharan, "Speckle-shearing interferometry: a simple optical system”, Appl. Opt. 14 (11), 2563 (1975). 
6. Y. Iwahashi, K. Iwata, and R. Nagata, "Single-aperture speckle shearing interferometry with a single grating", Appl. Opt. 23 (2), 247-249 (1984).

7. C. Joenathan and R. S. Sirohi, "Holographic gratings in speckle shearing interferometry", Appl. Opt. 24 (17), 2750-2751 (1985).

8. H. Rabal, R. Henao, R. Torroba, "Digital speckle pattern shearing interferometry using diffraction gratings", Optics Comm. 126, 191-196 (1996).

9. C. Joenathan, L. Bürkle, "Elecktronic speckle pattern shearing interferometer using holographic gratings", Opt. Eng. 36 (9), 2473-2477 (1997).

10. Emilia Mihaylova, Izabela Naydenova, Hosam Sherif, Suzanne Martin, Vincent Toal, "Application of photopolymer holographic gratings in electronic speckle pattern shearing interferometry", Proceedings of SPIE 5249, 318-326 (2003).

11. Emilia Mihaylova, Izabela Naydenova, Suzanne Martin, Vincent Toal, "Electronic speckle pattern shearing interferometer with a photopolymer holographic grating", Applied Optics 43 (12), 2439 - 2442 (2004).

12. S. Martin, P. Leclère, V. Toal, Y. Renotte and Y. Lion, "Characterisation of acrylamide-based photopolymer holographic recording material", Opt. Eng. 32 (12), 3942 - 3946 (1994).

13. J. C. Wyant, "Double frequency grating lateral shear interferometer", Appl. Opt. 12, 2057 - 2060 (1973).

14. Maurice P. Whelan, Coin Forno, Suzanne Martin, Feidhlim O'Neill, Vincent Toal, "Illumination systems using photopolymer gratings for spechle interferometry", Proceedings of "Interferometry '99" - International Conference on Optical Metrology, Techniques and Technologies, Warsaw, Poland, September $20^{\text {th }}-23^{\text {rd }}, 1-8$ (1999).

15. "EspiTest" Software, developed by Andreas Langhoff and Maurice Whelan (http://www.daedalussoft.com), Copyright $\odot 1998$ European Commission, DG - Joint Research Centre, Ispra, Italy. 